山्山FFANÇAISE

$\supset \mathrm{DE}$

$\stackrel{1=1}{\simeq}$ PÉDAGOGIE

\section{Revue française de pédagogie}

Recherches en éducation

182 | 2013

Connaissances et politiques d'éducation : quelles interactions?

\title{
Hiérarchie des savoirs et concurrences institutionnelles : la régulation des cartes régionales des formations professionnelles initiales
}

Knowledge prioritisation and institutional competitions: the regulation of local maps of initial training programmes

Hélène Buisson-Fenet et Éric Verdier

\section{(2) OpenEdition}

Édition électronique

URL : http://journals.openedition.org/rfp/3987

DOI : $10.4000 /$ rfp.3987

ISSN : 2105-2913

Éditeur

ENS Éditions

Édition imprimée

Date de publication : 28 août 2013

Pagination : $19-30$

ISSN : 0556-7807

Référence électronique

Hélène Buisson-Fenet et Éric Verdier, « Hiérarchie des savoirs et concurrences institutionnelles : la régulation des cartes régionales des formations professionnelles initiales », Revue française de pédagogie [En ligne], 182 | 2013, mis en ligne le 28 août 2016, consulté le 21 avril 2019. URL : http:// journals.openedition.org/rfp/3987 ; DOI : 10.4000/rfp.3987 


\title{
Hiérarchie des savoirs et concurrences institutionnelles : la régulation des cartes régionales des formations professionnelles initiales
}

\author{
Hélène Buisson-Fenet et Éric Verdier
}

Composante majeure de l'enseignement secondaire français, la formation professionnelle initiale a progressivement été structurée par des orientations volontaristes nationales et des demandes locales. Cette construction s'est accompagnée du développement d'outils de connaissance dont les cartes des formations sont une expression emblématique. Contrastant les configurations régionales de l'action publique qui leur donnent forme, cet article révèle les relations de concurrence et de coopération cognitives qui structurent la dynamique de l'action publique ; l'ambition de promouvoir une régulation polycentrée fortement adossée à une expertise régionale originale s'avère nettement déçue, en raison notamment d'un contrôle gestionnaire rapproché développé par les représentants de l'État en région. Cette technicisation de l'action publique, largement unilatérale, n'empêche pas l'outillage partenarial mis en place au titre des plans régionaux de produire des effets tangibles en matière de coordination.

Mots-clés (TESE) : formation et enseignement professionnels, formation initiale, politique en matière d'éducation, niveau régional.

Avec près de $45 \%$ d'une classe d'âge préparant un diplôme professionnel ou technologique en établissement scolaire, le modèle français de formation professionnelle initiale est marqué par la "scolarisation " des formations. Progressivement mise en place (Suteau, 1999), cette configuration résulte d'abord d'une histoire longue qui, dans l'après-guerre en particulier, voit se conjuguer deux processus : d'une part, l'interventionnisme de l'État planificateur et la réticence du patronat à assumer les coûts de la formation initiale, qui se traduisent dans les années 1960 par l'intégration de l'enseignement technique au "système " scolaire (Troger, 2008) ; d'autre part, l'expression de demandes sociales et économiques locales inscrites dans des spécificités productives des territoires parfois fort anciennes et qui se sont matérialisées dans une offre structurée (Caro \& Hillau, 1997). Mais ce développement est aussi le fruit plus récent d'orientations volontaristes nationales, dont est emblématique la création, en 1985, des baccalauréats professionnels.

Cette construction s'est accompagnée du développement d'outils de connaissance fortement stabilisés. Les cartes de la formation professionnelle initiale sont au nombre de ces instruments, issues de concertations entre les trois acteurs collectifs principaux que sont les services académiques, les services régionaux de formation professionnelle et les représentants des branches professionnelles. En examinant à la fois 
leur élaboration au long cours et en contrastant les configurations régionales de l'action publique qui leur donnent forme dans les années 2000 , on révèle les relations de concurrence et de coopération cognitives qui structurent la dynamique de l'action publique.

Aussi, les cartes des formations professionnelles initiales auraient évolué d'un statut d'équipement de pouvoir définissant un rapport social de domination entre la puissance publique et ses destinataires (Fourquet \& Murard, 1976) à un rôle d'outil de régulation, c'est-à-dire à un objet technique dont la production et l'usage structurent le positionnement des acteurs. Une première hypothèse soutient que l'instrument " carte de formation " n'existe pas isolément, mais s'inscrit dans un dispositif d'arbitrages dont la géométrie varie selon le contexte régional et le jeu d'acteurs qui s'y donne à voir. On postule aussi que son usage s'intègre dans un travail de coopérations versus des concurrences de logiques professionnelles et organisationnelles diversifiées suivant la configuration régionale en cause. tualisation de l'action publique, telle qu'elle existait dans le champ de la formation continue avant même la loi fondatrice du 16 juillet 1971 qui créa l'obligation annuelle pour tout employeur d'affecter une fraction de la masse salariale au financement de la formation des salariés ${ }^{1}$. Ce gouvernement par contrat (Gaudin, 1999) a favorisé l'émergence de nouvelles scènes collaboratives en région (Jobert, 2002), notamment en ouvrant la régulation des politiques régionales de formation initiale et continue à la participation des acteurs économiques et sociaux. À la fin des années 1980, cette ouverture s'est appuyée sur une capacité d'expertise de plus en plus équipée, en particulier sous la forme d'Observatoires régionaux emploi formation (OREF) ; depuis le milieu des années 2000 se dessine une réaffirmation de l'intervention centrale dans la régulation des places, fondée notamment sur des instruments gestionnaires issus des statistiques académiques de démographie scolaire. Peut-on pour autant en déduire que l'on retrouve la place historique des savoirs et des instruments d'État dans cette régulation sectorielle?

\section{La prédominance historique des savoirs d'État dans la constitution des cartes des formations professionnelles}

\section{DÉCENTRALISATION ET FORMATION PROFESSIONNELLE : L'AMBITION DÉÇUE D'UNE RÉGULATION SAVANTE POLYCENTRÉE ?}

En matière de formation initiale, les transferts de compétences aux Régions ont impulsé une contracgouvernementale se traduit par une construction articulée des politiques de formation et d'emploi
Dans les années 1960, la «mise en Plan » de l'action

Dans le cadre du projet européen Knowledge and Policy (Know\&Pol) piloté par B. Delvaux et É. Mangez (Université catholique de Louvain), la démarche s'est appuyée sur différents corpus :

1. Les plans, schémas prévisionnels et contrats d'objectifs régionaux configurant la relation formationemploi et son évolution souhaitable par territoires et branches professionnelles (notamment la production des Observatoires régionaux emploi formation [OREF]).

2. Les bases de données académiques retraçant la cartographie évolutive des formations initiales.

3. Une quarantaine d'entretiens approfondis (certains renouvelés jusqu'à trois fois pour actualiser les données dans une conjoncture de réforme du baccalauréat professionnel), menés auprès de responsables des services des conseils régionaux et des rectorats, de membres des corps d'inspection de l'Éducation nationale, de directeurs d'établissements de formation, de représentants des milieux professionnels (en particulier la branche BTP) engagés dans le travail de cartographie.

Les régions Provence-Alpes-Côte d'Azur (PACA) et Rhône-Alpes ont été choisies à la fois en raison de leur importance économique et démographique (deuxième et troisième après l'île-de-France) et pour leurs trajectoires politiques assez proches: depuis 2004, l'une et l'autre sont gouvernées par une majorité de " gauche plurielle "; elles ont connu auparavant une mandature en majorité relative avec, en Rhône-Alpes, un exécutif de centre droit et en PACA, un président socialiste, l'un et l'autre devant composer avec son opposition. Ces points communs présentent l'avantage de mieux faire ressortir les spécificités régionales en matière de relations formation-emploi, sachant qu'il a fallu aussi tenir compte (comme on le verra dans la deuxième partie) de particularismes académiques, chaque région comptant deux rectorats. 
(Tanguy, 2002), alors que, tendanciellement, elles sont conçues de manière distincte, au point que la formation professionnelle relève de deux départements ministériels ${ }^{2}$. Cette planification indicative s'appuie sur de puissantes technologies cognitives : l'élaboration au cours du Ve Plan (1966-1970) de la nomenclature interministérielle des niveaux de formation consistant à classer les individus en fonction de l'emploi occupé, lui-même étalonné selon le niveau d'études « correspondant "; la création en 1970 du Centre d'études et de recherches sur les qualifications (CEREQ) et, progressivement, de l'observatoire national des entrées dans la vie active, ou encore la fabrication, sous l'égide de I'INSEE, de bilans prévisionnels formation-emploi (INSEE, 1976). La montée en puissance de ces dispositifs marque d'abord l'emprise techniciste d'une administration " experte "3 (Fourquet, 1983). Au-delà, se diffuse une conception prévisionnelle et rationaliste de l'action publique, destinée notamment à mesurer et à répartir les flux à scolariser. L'approfondissement de la crise de l'emploi tout au long des années 1970 remet en cause cette approche (Affichard, 1976) et favorise le passage à une prospective plus compréhensive, mieux à même de saisir les tendances sociétales (Freyssinet, 1991). De ces deux étapes résulte, du seul côté de l'Éducation nationale, un déploiement de dispositifs que l'on peut distinguer selon les niveaux géographiques, bien qu'il y ait imbrication des rôles : au niveau national, le ministre fixe les grandes orientations de la politique éducative, les Commissions professionnelles consultatives (CPC) définissent les contenus des diplômes tandis que, en relation avec les divers corps d'inspection, l'inspection générale accompagne et vérifie l'application des textes ; au niveau académique, le recteur fixe les orientations spécifiques de l'académie, secondé par les responsables des directions fonctionnelles (le délégué académique à l'enseignement technique [DAET], le chef du service d'information et d'orientation [CSAIO], le chef du service statistique, le délégué académique à la formation continue [DAFCO]), tandis que les inspecteurs, chargés notamment des questions pédagogiques, assurent les relations entre le rectorat et les établissements ; au niveau local, le chef d'établissement et l'équipe pédagogique organisent les enseignements et formulent des propositions d'évolution de la carte.

Cette configuration institutionnelle et cognitive a installé des routines nationales et sectorielles d'autant plus puissantes qu'elles reposent sur le déploiement de corps d'inspection influents et de services académiques aux solides compétences techniques (Pons, 2012). Dans le cours d'une décentralisation qui attribue toujours au recteur le soin d'arrêter la carte des formations professionnelles et technologiques sous statut scolaire (la carte de l'apprentissage revenant au président du conseil régional), les décisions d'ouverture ou de fermeture de sections préparant à tel ou tel diplôme de formation professionnelle (du CAP au BTS) ajustent annuellement la structure pédagogique des établissements, en référence à différents paramètres que renseignent quatre séries d'indicateurs : la capacité d'accueil, le taux d'attractivité (rapport entre le nombre de vœux prioritaires d'orientation et la capacité d'accueil), le taux d'accueil (rapport entre le nombre de places et le nombre de vœux 1), le remplissage (rapport entre le nombre de présents à la rentrée et le nombre de places). Si les services rectoraux fournissent des statistiques synthétisées à différents niveaux à partir des applications informatiques que les établissements renseignent, les inspecteurs réalisent des visites de terrain à l'issue desquelles ils rédigent des "notes d'observation ", s'appuyant sur les trois techniques d'enquête que sont l'analyse de documents (notamment l'interprétation des indicateurs de pilotage de l'établissement), l'observation (notamment la visite des ateliers) et l'entretien (avec le chef d'établissement, le chef de travaux, quelques enseignants). Cette pluralité des données, tant quantitatives que qualitatives, assure à la DAET un positionnement cognitif particulièrement solide face à ses interlocuteurs, qu'il s'agisse des organismes professionnels ou de la Région, au risque pour ces derniers d'être confinés dans des consultations assez formelles.

\section{Au cœur de l'action publique territoriale, l'émergence et le développement d'une expertise régionale}

Avec le contrat de plan État-Région institué par la loi du 29 juillet 1982, le schéma prévisionnel des formations (loi du 22 juillet 1983) et celui de l'apprentissage (loi du 23 juillet 1987), il revient aux Régions de transposer à leur échelle d'action la figure de «l'État ingénieur » (Desrosières, 2003), dont on attend qu'elle retrouve, grâce aux facilités coordonnatrices de la "proximité ", une part de pertinence politique et technique très largement perdue au niveau national.

En 1993, la dévolution aux Régions de la fonction d'animation de l'action publique en matière de formation des jeunes s'est traduite par l'invention et l'usage d'un instrument privilégié, le Plan régional de développement de la formation professionnelle (PRDF) ${ }^{4}$. Défini dans le code de l'éducation, son objet tient à « la programmation à moyen terme d'une offre régionale 
de formation de nature à permettre un développement cohérent de l'ensemble des filières de formation et qui sache prendre en compte les réalités économiques régionales et les besoins des jeunes de manière à leur assurer les meilleures chances d'accès à l'emploi "; il est ainsi en charge de la coordination de la formation sous statut scolaire, de l'apprentissage et de la formation par alternance. En outre, comme le montre l'exemple significatif de la région PACA, le PRDF doit s'efforcer de « mettre en cohérence l'effort de formation professionnelle avec d'autres politiques régionales définies elles aussi dans des documents d'orientation et de programmation votés par le Conseil régional [comme] le schéma régional d'aménagement du territoire (SRADT), le schéma régional de développement économique (SRDE), le plan régional pour l'emploi (PRE), le schéma régional de l'enseignement supérieur et de la recherche (SRES) [...] $»^{5}$. Est en jeu l'élaboration d'un projet régional destiné à couvrir non seulement la relation formation-emploi mais plus largement la stratégie régionale de développement économique et social. Comme dans le cas de la planification française des Trente Glorieuses, cette construction politique et technique n'est pas dissociable d'une intense activité de régulation sociale qui donne lieu à un écheveau complexe de négociations et de consultations. Aussi le PRDF peut-il être qualifié de « méta-instrument » de l'action publique régionale de formation professionnelle (Verdier \& Vion, 2005).

En surplomb de la régulation des places de formation, tout PRDF est appelé à positionner la stratégie régionale en matière de qualifications vis-à-vis de trois clés d'entrée : les attentes individuelles, les branches et les territoires, chacune exprimant une « demande " à l'égard des appareils de formation initiale et continue. La première consiste à répondre aux projets et aux vœux d'orientation formulés par les jeunes et leurs familles (Berthet, Dechezelles, Gain et al., 2010). La deuxième vise à répondre aux attentes des entreprises en termes de disponibilité des qualifications en quantité comme en qualité. La dernière concerne le développement des territoires en vue de répondre à une double préoccupation : l'égalité des chances d'accès à la formation quel que soit le lieu de résidence et la mobilisation de la formation au service de projets de développement territorial (" pays » ou communautés de communes par exemple). Dès la première génération des PRDF, au milieu des années 1990, la prise en compte de ces trois types de demande a conduit les pouvoirs publics à afficher leur volonté de limiter le poids des logiques d'offre, c'est-à-dire des dynamiques endogènes des appareils de formation (Bel, Gérard-Varet \& Verdier, 2000).
Cette activité prévisionnelle a engendré une demande importante de chiffres en vue d'établir des " diagnostics partagés » et a contribué à valoriser les missions des OREF, créés formellement en 1987 et financés par les contrats de plan État-Région (Healy \& Verdier, 2010). L'approche par branches de la relation régionale formation-emploi a été privilégiée pour deux raisons : elle correspond à la construction historique de la formation professionnelle en France, et la régulation des formations continues et en alternance est fortement structurée par la négociation nationale de branche (Méhaut \& Verdier, 2009). Aussi l'activité initiale des OREF a-t-elle privilégié des diagnostics sectoriels emploi-formation mobilisant à la fois les travaux des antennes régionales de I'INSEE et les compétences des services statistiques ministériels (Pons, 2012). Très tôt par exemple, l'OREF RhôneAlpes (OREFRA) monte une "banque de données des ressources humaines " croisant qualifications et secteurs ; cette représentation statistique, dont l'outil majeur est constitué d'une nomenclature de familles professionnelles articulant spécialités de formation et spécialités d'emploi, favorise l'émergence d'un langage commun aux acteurs sociaux et publics ${ }^{6}$. Plus tardivement, à la fin des années 1990, l'observatoire régional des métiers de PACA propose un appareillage sophistiqué de diagnostics sectoriels des qualifications. Progressivement, notamment pour rendre compte des mobilités des personnes, ces OREF développent des travaux relevant des deux autres clés d'entrée que constituent les territoires (infrarégionaux) et les trajectoires individuelles. En outre, dans une perspective de rapprochement de la connaissance et de l'action, les OREF promeuvent des démarches d'accompagnement des acteurs au point de modifier sensiblement leur positionnement institutionnel : non seulement ils passent de la figure du dossier statistique descriptif à celle du diagnostic visant à dégager des enjeux stratégiques pour l'action publique régionale (années 1990), mais, en outre, ils favorisent désormais l'appropriation de ces « produits » statistiques par leurs utilisateurs, qu'il s'agisse de regroupements d'employeurs, de porteurs de projets de développement territorial, de responsables de dispositifs de formation, etc. Ainsi les OREF ambitionnent-ils de devenir l'institution d'expertise légitime en région sur les enjeux articulant formation, emploi et développement économique. 


\section{« Retour de l'État » ou contrôle gestionnaire rapproché ?}

Si les progrès des observatoires soutiennent aujourd'hui mieux qu'hier la construction d'une «localité » (Desrosières, 2008) en tant que représentation légitime d'une entité régionale dotée d'une stratégie de développement spécifique, encore faut-il que celleci parvienne à s'imposer techniquement et politiquement. Or l'observation séquentielle de la régulation des cartes des formations professionnelles rend compte de la difficulté concrète à recomposer les hiérarchies de savoirs et, partant, les rapports de force politiques institués.

Alors que l'élaboration des PRDF a suscité un lourd travail statistique de bilan et de diagnostic destiné à équiper la prospective des qualifications, l'analyse des procédures de révision donne à voir une forte “ dépendance de sentier » à l'égard d'une régulation propre au secteur de l'éducation. La lettre de cadrage de la procédure de révision de la carte des formations professionnelles initiales, cosignée par le recteur et le président du conseil régional, fait toujours référence aux objectifs généraux de la politique nationale en matière éducative ; les inspecteurs s'appuient de leur côté sur une connaissance très fine des établissements, des contenus de formation offerts et, souvent, des qualifications demandées par les entreprises locales ; les conseillers d'orientation se focalisent sur les ressorts de la demande sociale de formation en mobilisant les statistiques académiques sur les vœux des élèves et leurs cheminements entre les établissements de la zone. Réunissant les différents avis et ceux des branches professionnelles, le délégué académique à l'enseignement technique (DAET) transmet au recteur un avis de synthèse sur l'opportunité d'ouvrir ou de fermer telle ou telle section. II revient à la direction de l'organisation scolaire du rectorat d'intégrer les incidences de l'évolution de la carte sur les postes d'enseignants, d'autant que d'ultimes ajustements peuvent avoir lieu à l'issue des commissions paritaires qui associent administration académique et syndicats. Au final, le caractère largement interne à l'Éducation nationale du processus atteste la place prééminente qu'occupent les autorités académiques, par delà les négociations et échanges avec les services de la Région dont la marge de manœuvre décisionnelle s'avère singulièrement réduite, ce qui « fausse le jeu » comme l'exprime en entretien un membre de la direction de la formation de la Région Rhône-Alpes.

Cette asymétrie est encore aggravée par la faible implication des branches professionnelles. Certes, formellement, leur consultation est d'autant plus importante qu'une série de conventions lie les rectorats à certaines d'entre elles et qu'en outre, l'État - rectorats et préfecture de région -, le conseil régional et les branches professionnelles les plus importantes ont conclu, à compter de 1987, des contrats d'objectifs censés favoriser, au niveau sectoriel, une gestion « cohérente » des formations professionnelles initiales et continues. Mais en réalité, cette procédure s'avère faiblement outillée. Peu d'analyses statistiques sont fournies pour étayer les avis que les branches formulent en réponse à la lettre que leur adresse le recteur pour solliciter leurs points de vue sur le bien-fondé des demandes émises par les établissements dans les spécialités professionnelles qui les concernent. Cet outillage minimal donne lieu à des réponses techniquement très hétérogènes, reflétant un équipement très variable des branches, du moins pour celles qui répondent - ce qui, en PACA, n'est le cas que de la moitié d'entre elles. Tout se passe comme si elles préféraient garantir leur pouvoir d'influence sur les formations en alternance (contrats de professionnalisation mais aussi apprentissage) en restant prudemment extérieures au processus (Méhaut \& Verdier, 2009). Cette contractualisation par branches s'avère incapable de contribuer à la mise en cohérence des cartes des formations professionnelles initiales et continues : les conventions d'application qui devaient encadrer leur évolution annuelle n'ont pas la régularité nécessaire ; l'extension du champ de ces contrats aux questions d'emploi et de travail se traduit par une certaine dilution des enjeux; enfin, la place plus large donnée dans certaines Régions aux organisations interprofessionnelles (en Rhône-Alpes notamment), dans d'autres aux confédérations syndicales (en PACA par exemple), conduit au désengagement de certaines branches.

La modestie des évolutions constatées au regard de la lourdeur excessive de la machinerie procédurale est régulièrement dénoncée (Mission d'audit de modernisation, 2006). En outre, les contingences propres au champ politique réactivent des clivages et des asymétries peu favorables à une régulation conjointe qui aille au-delà de l'affirmation de grands principes. Se succèdent depuis 2005 des réformes élaborées sans grande concertation avec les collectivités territoriales, alors même qu'elles concernaient très directement les compétences transférées à ces dernières : «l'apprentissage junior » accessible à 14 ans du Plan d'urgence pour l'emploi (2006) fera même l'objet d'un refus de l'ensemble des Régions. Ces dernières vont promouvoir, de manière plus ou moins véhémente, " un service public régional de la formation professionnelle et de l'apprentissage ». Inscrite dans une rhétorique avant 
tout politique, cette orientation est adoptée sans guère de concertation avec les services de l'État, pourtant en charge du contrôle pédagogique et social de l'apprentissage et du contrôle réglementaire de la formation professionnelle continue?.

Ce dissensus politique croissant entre l'État et les Régions conduit chaque partenaire à réguler de manière autonome le dispositif de formation dont il a juridiquement la responsabilité. À l'ère d'une nouvelle régulation "par les résultats", les recteurs doivent mettre sur pied des projets académiques visant à décliner les programmes nationaux d'action et de performance de la loi organique relative aux lois des finances (LOLF), en exerçant pleinement leurs prérogatives institutionnelles. Pour un rectorat, affirmer son autonomie est relativement peu coûteux en termes organisationnels puisqu'il dispose des services compétents (corps d'inspection, DAET, services de l'orientation) qu'il remobilise autour de ses propres orientations politiques dans le champ de ses responsabilités directes : ainsi, à rebours du décloisonnement institutionnel visé par le PRDF, se reconstituent les avantages d'une politique sectorielle (les formations de l'Éducation nationale) partiellement affranchie des pesanteurs de partenariats multi-acteurs (avec les différentes collectivités territoriales, les autres offreurs de formation, les partenaires sociaux, etc.), souvent plus subis que choisis. Côté Région, les conséquences sont nettement plus négatives compte tenu de capacités d'expertise beaucoup plus légères. Enfin, si les justifications gestionnaires ne sont pas nouvelles, elles sont depuis 2007 une référence croissante des logiques d'action. D'un côté, la Région, qui estime assurer "le gîte et le couvert », reproche régulièrement à l'État les limites de sa prospective :

"Quand on se ballade dans les ateliers, y'a jamais personne ou très peu de monde, donc on trouve que nous, du point de vue de la conception des bâtiments et des espaces et bien c'est quand même du gaspillage, et que l'Éducation nationale de ce point de vue là, et bien on voit bien que c'est pas elle qui paie. " (directeur général adjoint, Région RhôneAlpes).

Mais le référentiel gestionnaire n'est pas l'exclusivité du financeur : les directions de l'organisation scolaire (DOS) des rectorats, qui arbitrent le nombre de divisions par spécialités de formation, insistent sur les économies d'échelle qu'une réforme comme celle du baccalauréat professionnel, qui fait passer la durée du cursus de quatre à trois ans, permet d'envisager. Dans un tel contexte, l'habileté à justifier l'usage des ressources devient un savoir de plus en plus indispensable à qui veut concilier des objectifs " ambitieux » en matière de formation et une gestion «rationalisée » des moyens.

\section{DES CONFIGURATIONS RÉGULATION- CONNAISSANCES TERRITORIALEMENT DIFFÉRENCIÉES}

Par delà une commune autonomie de l'offre de formation, il s'avère que l'équilibre des forces entre acteurs territoriaux et représentants de l'État diffère sensiblement d'une période à l'autre et d'une région à l'autre. Dans les années 2000, les PRDF de PACA et de Rhône-Alpes placent l'élaboration en commun et le partage de connaissances diagnostiques de la relation formation-emploi au cœur de la régulation des cartes des formations professionnelles. Après d'intenses mobilisations par ou autour de ces diagnostics se réaffirment des régulations autonomes (rectorats versus Régions) des dispositifs de formation initiale qui affaiblissent la portée de ces investissements cognitifs. Dans ce contexte, l'établissement (le lycée) devient le pivot privilégié de la production de connaissances utiles et légitimes pour la régulation des cartes.

\section{Le début des années 2000 : les connaissances au cœur de l'action publique}

En PACA, l'adoption en décembre 2002 du PRDF par l'assemblée régionale est le point d'orgue d'une année de consultations et d'auditions : ce plan affiche une volonté générale de coopération plus qu'il ne détaille les opérations à réaliser. Afin de favoriser l'intéressement des parties prenantes à la construction du bien commun, la Région propose à l'État de s'engager, pour la période 2002-2007, sur « six orientations pour mieux répondre aux enjeux économiques et sociaux régionaux » dont, classiquement, le renforcement « de l'égalité des chances pour l'accès aux savoirs et à une première qualification ".

Dès la phase d'élaboration de ce PRDF « contractualisé " se développe une dialectique de renforcements réciproques entre, d'un côté, l'approfondissement des partenariats et, de l'autre, le développement de l'institution « savante " que cherche à être l'observatoire régional emploi formation. Le plan érige en effet la connaissance diagnostique en une ressource indispensable au pilotage de l'action publique : y figurent " des outils de diagnostic de la demande sociale, de la demande économique et de l'offre de formation; des cahiers prospectifs par grandes familles 
professionnelles; des outils d'observation des parcours " sur le marché du travail. La préparation du schéma prévisionnel des formations donne lieu à la réalisation de deux diagnostics fouillés : le premier, territorial, caractérise les enjeux en matière d'évolution de l'offre de formation sur l'ensemble des bassins d'éducation et de formation ; le second, organisé par domaines de formation, vise à identifier les enjeux liés au renouvellement des qualifications. L'observatoire connaît dès lors un très fort développement, passant de quatre collaborateurs à une quinzaine. L'assise acquise est telle que, malgré l'affaiblissement ultérieur de «l'action publique savante » qui l'a porté, cet observatoire continuera à conforter son ancrage institutionnel et sa légitimité technique.

En Rhône-Alpes, le tournant est pris en 2006 avec un PRDF qui diffère radicalement des précédents : l'exécutif opte pour un document purement procédural qui veut être un "véritable outil de pilotage opérationnel et de concertation pour faire évoluer l'offre de formation, en produisant à la fois des éléments de diagnostic et de programmation pluriannuelle et un dispositif permanent d'élaboration par l'ensemble des partenaires des conventions annuelles d'application [du PRDF] ". Sa gouvernance à la fois cognitive et politique repose sur trois groupes de travail, chargés « de compléter les analyses prospectives de l'emploi et de la formation réalisées au niveau national ». Chaque groupe est piloté par l'un des partenaires de la régulation tripartite : le groupe Publics et parcours par l'État, le groupe Secteurs économiques est confié aux partenaires sociaux et le groupe Territoires à un élu régional. Un comité de pilotage est chargé de faire la synthèse des travaux des trois groupes et d'établir des propositions d'orientation et de formation, soumises ensuite à la commission du PRDF.

Cette démarche, qui imbrique choix politiques et élaboration de connaissances idoines, s'appuie sur un groupement d'intérêt public, le Pôle Rhône-Alpes de l'orientation (PRAO), créé par les acteurs du tripartisme régional en 2004. Devenu une composante technique de ce pôle qui fédère 21 "réseaux et services d'orientation ", l'OREFRA et ses collaborateurs sont appelés à informer l'action régionale et à la rendre lisible. II s'agit de contribuer à l'organisation des parcours individuels dans le cadre d'une " orientation tout au long de la vie " (CESR Rhône-Alpes, 2004). Cette perspective vise à fournir des services opérationnels aux acteurs de la relation formation-emploi, à proposer une « information pertinente et lisible » pour les professionnels de l'orientation, à " apporter un appui aux décideurs » et, enfin, à développer sur l'ensemble du territoire régional l'accès à la validation des acquis de l'expérience (VAE). Ce PRAO-OREF propose une approche des territoires très fine, à finalité opérationnelle : au niveau des 27 zones territoriales (comme de chacun des départements et de la Région), la base de données se structure autour de questions concrètes pour permettre à l'utilisateur de forger son propre diagnostic ${ }^{8}$.

\section{Depuis 2006 : l'essoufflement d'une régulation conjointe fondée sur une expertise partagée}

Après la phase de mobilisation autour de ces diagnostics partagés se réaffirment dans les deux régions des régulations autonomes des dispositifs de formation initiale. Plusieurs raisons se conjuguent pour saper l'effectivité du bel édifice politico-cognitif : à l'incapacité de la contractualisation par branches à mettre en cohérence les différentes filières de formation professionnelle s'ajoutent : en PACA, l'élaboration avortée du schéma prévisionnel des formations initiales qui prive le PRDF de son outillage technique et institutionnel indispensable à son effectivité ${ }^{\text {; }}$; en Rhône-Alpes, le faible apport de certains travaux diagnostiques et prospectifs (parcours de formation et territoires) à la programmation régionale de la formation. Dans le premier cas, le processus a pâti d'une participation irrégulière des représentants du service public d'éducation, dans le second, il s'est avéré très difficile d'opérationnaliser "la place centrale donnée aux territoires » dans le PRDF. Malgré la multiplication des concertations, les PRDF peinent à se doter d'une traduction opérationnelle probante. Dès lors, la voie est ouverte à des démarches plus unilatérales que concertées ou négociées, donnant prise aux logiques propres des rectorats, qui se distinguent parfois au sein d'une même Région.

Ainsi, en Rhône-Alpes, les conditions d'application de la réforme consistant à faire préparer le baccalauréat professionnel en trois ans - au lieu de quatre varient au point de rendre compte du poids prééminent des contextes gestionnaires des rectorats sur les contextes politiques et économiques propres à la Région. Deux dimensions principales expliquent ces variations : la gestion des temporalités et la dotation en postes de l'académie. Les méthodes adoptées à Lyon et à Grenoble diffèrent, en particulier au regard du temps laissé à la discussion avec les équipes du conseil régional. Ainsi, côté grenoblois, l'accent mis sur l'élaboration préalable d'un diagnostic commun sur l'état des diverses spécialités ménage un temps conséquent pour que soient prises en compte les 
contraintes pédagogiques, techniques et gestionnaires de chaque partenaire. La généralisation de la méthode s'opère sans heurts. Le recteur de Lyon se centre, quant à lui, sur l'administration rapide et unilatérale de la potion amère que sont les suppressions de postes dans un contexte de fort recul démographique. En raison du cumul de suppressions de sections préparant à tel ou tel diplôme, certains lycées voient leurs effectifs chuter à moins de 200 élèves, leur attractivité déjà déclinante se dégrader encore plus et leurs taux d'encadrement s'élever au risque de justifier de nouvelles suppressions de postes au nom de la bonne et équitable gestion. C'est pour conjurer de tels aléas que, dans sa délibération relative au projet de convention 2009 du PRDF, le conseil régional de Rhône-Alpes demande " au Recteur [de Lyon] le réexamen de [ses] propositions concernant 10 établissements, en raison de l'insuffisance des ouvertures envisagées pour préserver [leurs] capacités d'accueil à l'horizon 2012 ", en soulignant que ces établissements sont situés sur des territoires eux-mêmes fragilisés sur le plan économique et social - la Loire et les banlieues lyonnaises.

Prenant acte de la «panne » des dispositifs partenariaux au moment de l'élaboration du projet académique, le recteur d'Aix-Marseille réaffirme la légitimité prééminente de ses services en matière de carte des formations professionnelles scolaires. À compter de 2005 et au titre de la LOLF, la modernisation de la gestion académique est portée par un programme intitulé La route haute vers la réussite scolaire, qui rappelle certes la nécessité de respecter les " cadres structurants " définis avec le conseil régional, mais dont la cohérence est clairement attachée à la réalisation de «programmes de performance » de l'académie impliquant les projets des établissements publics locaux d'enseignement (EPLE). Par ailleurs, la règle qui prévaut en matière d'ouverture de sections repose désormais sur la technique du " gage » : toute ouverture d'une section dans un lycée doit être gagée par une fermeture dans le même établissement, comme l'illustre la négociation suivante :

Proviseur : Nous avons une deuxième proposition, un CAP «transport par câble » [...] il y a des possibilités de stages dans les stations de ski proches.

Inspecteur : Le président du BTP 05 est favorable pour les Hautes-Alpes.

Proviseur : C'est en cohérence avec les spécificités professionnelles locales.

Recteur : Le financement de ce CAP est prévu comment?
Proviseur : On va transformer le cycle BEP-Bac pro 4 ans en Bac pro 3 ans, donc on gagne un an.

Recteur : Oui, mais dans trois ans... Le dossier est intéressant, mais il faut trouver 16 fois deux heures, et vous n'avez pas de gage autre que virtuel à proposer.

\section{L'EPLE AU CENTRE DE LA CONCURRENCE DES EXPERTISES PROFESSIONNELLES}

Avec l'affaiblissement de la production diagnostique adossée aux seules sources statistiques, le niveau « établissement » et la culture locale qui lui est endogène, déjà supports de savoirs " expérientiels ", reviennent au cœur de la régulation des cartes des formations. L'offre du bassin, l'évolution locale de l'emploi et le nombre d'entreprises susceptibles d'accueillir les élèves en stage sont autant de données partagées par les inspecteurs et par les chargés de mission de la collectivité régionale. Elles peuvent être cadrées par des labellisations telles que celles associées au dispositif "lycées des métiers " dont les critères renvoient notamment à la " cohérence » de l'offre de formation, ou à la "pertinence des partenariats " avec les collectivités et les milieux professionnels, soit des attributs que seule une évaluation subjective des ressources de proximité saurait renseigner.

À ce titre, deux rôles professionnels se trouvent particulièrement sollicités par la régulation. D'un côté, les chefs d'établissement élaborent un « rapport général d'opportunité » qui cadre les demandes au regard de l'attractivité potentielle des nouvelles formations en intégrant l'évolution de la démographie lycéenne, les choix d'orientation des jeunes, les perspectives locales en matière d'emploi, les possibilités de stages, les avis des entreprises concernées, etc. De l'autre, les inspecteurs de l'enseignement technique construisent leur expertise en s'appuyant à la fois sur des données administratives qu'ils utilisent comme indicateurs et sur des diagnostics issus de visites de terrain. Ils occupent une place nodale dans les relations bilatérales entre les acteurs de la relation formation-emploi, à la fois impliqués dans la formulation des attentes des branches professionnelles et à l'interface entre les différents niveaux des services de l'État, tout en étant régulièrement mobilisés par les commissions emploiformation du conseil régional. Au-delà de l'évaluation des pratiques pédagogiques des enseignants, leur rôle consiste à arbitrer les intérêts souvent divergents des acteurs de proximité de la formation et ceux des représentants du monde professionnel, sachant que ce 
dernier est tiraillé entre les attentes hétérogènes des divers sous-groupes de métiers d'une même filière.

Des tensions opposent fréquemment les inspecteurs aux chefs d'établissement. Les premiers défendent une vision "réaliste ", au sens où elle prétend intégrer les contraintes gestionnaires, mais aussi " synoptique ", prenant en compte l'échelle du bassin de formation et de son réseau d'établissements ; les seconds épousent davantage une logique de projet centrée sur leur seul établissement, parfois déconnectée des coûts et des perspectives d'insertion :

"Chaque proviseur a décidé de continuer, de garder sa section, [...] alors ça fait cinq plateaux de 15 élèves, pour qui il va falloir une machine de coupe à plat, sept mètres de long, plus de deux tonnes [...] alors au lieu d'équiper un plateau de 30 élèves, là on a équipé cinq sur Marseille, deux sur Avignon. II n'y a pas une entreprise sur Avignon qui fait ça, hein, alors là placer les élèves en stages voyez le rouge là ? [...] en plus les proviseurs font leur pub, on regarde leur plaquette [...]. Moi j'arrive derrière, "attention on enseigne ça", je suis sans arrêt en train de verrouiller. " (inspecteur de l'Éducation nationale de l'enseignement technique, académie d'Aix-Marseille).

Le travail concret de cartographie des formations donne ainsi à voir des arbitrages tactiques, révélateurs d'une série de clivages de rôles qui articulent la division du travail cognitif attachée aux choix politiques avec des concurrences professionnelles, dont le niveau " établissement » rend particulièrement compte.

\section{CONCLUSION}

Dans un ouvrage-bilan sur les vingt premières années de la décentralisation, M. Bel et L. Dubouchet constatent en 2004 : " l'État ne parvient pas à sommer ses propres services de faire réussir la décentralisation en l'accompagnant au lieu de camper sur leurs propres prérogatives ". L'examen des productions cognitives et des dispositifs experts à l'œuvre dans deux régions nous semble indiquer combien il est nécessaire de nuancer ce propos en prenant en compte des arènes d'action publique contrastées. Certes les conseils régionaux ne sont pas les " grands coordonnateurs » que la loi de décembre 1993 espérait voir advenir. Néanmoins, le cadrage des pratiques par les outils assure une coordination minimale qui évite de trop sérieux débordements. La technicisation de l'action publique en matière de formation profession- nelle initiale ne se traduit pas tant par une rationalisation des investissements que par une recherche de légitimation qui oblige les partenaires les uns envers les autres, et qui, par un effet de cliquet, assure davantage la continuité des décisions par delà l'incertitude des mandats.

En premier lieu, les équipes des différents acteurs publics en charge des cartes des formations professionnelles initiales s'accordent pour adhérer à une approche non " adéquationniste " de la relation formation-emploi : le devenir professionnel des jeunes ne saurait être strictement limité à la spécialité de formation suivie en formation initiale. Aussi la nécessité de fermer ici ou là une section préparant à telle spécialité au prétexte que les débouchés spécifiques faiblissent est relativisée : dès lors, le jugement en situation gagne en légitimité et valorise les savoirs d'expérience forgés par des experts professionnels tels que les inspecteurs. Par exemple, seuls les inspecteurs seront en mesure de proposer un compromis entre les contraintes de la démographie scolaire et la préservation « raisonnable » de structures pédagogiques existantes, en faisant valoir que subsistent des "viviers " d'élèves suffisants pour justifier le maintien d'une partie au moins des postes budgétaires concernés. Une seconde dimension d'ordre paradigmatique a trait à un principe d'égalité d'accès à la formation, notamment territoriale : à rebours de l'application mécanique de critères de gestion fondés sur des taux de remplissage des formations, on maintiendra des formations comptant un nombre important de places vacantes au nom d'impératifs d'aménagement du territoire.

Par ailleurs, l'outillage mis en place dans le cadre de la démarche partenariale liée au PRDF produit des effets tangibles en matière de coordination, par delà une coopération affaiblie depuis 2005. La mise au point de calendriers convergents, les productions des OREF, les bases de données et les indicateurs communs cadrent la régulation des cartes pour faire en sorte que les désaccords demeurent des exceptions. Cette configuration favorise des évolutions incrémentales des dispositifs de formation en confortant la très grande dépendance de sentier de ces derniers. Elle est pourtant régulièrement dénoncée en raison du conservatisme des intérêts qu'elle exprimerait et de la lourdeur excessive d'une machinerie procédurale imposante au regard de la modestie des évolutions constatées. Aussi, paradoxalement, s'impose peu à peu l'idée selon laquelle seules des réformes venues d'en haut seraient de nature à produire des changements significatifs. 
Les justifications managériales, sans être nouvelles, s'affirment et se diffusent dès lors de plus en plus, les Régions n'échappant d'ailleurs plus à ce mouvement, tenues qu'elles sont par des dotations budgétaires serrées. Dans un tel contexte, la justification de choix dérogatoires et a fortiori discrétionnaires monte en sophistication : la localisation d'un projet de lycée doit désormais faire état du nécessaire vivier d'élèves pour que la décision de création soit opposable ; le maintien de postes doit à présent faire valoir de fortes exigences territoriales en matière d'intégra- tion d'élèves handicapés. La politique revient alors au centre des ajustements, avec la nécessité maintenue de justifier pourquoi les décisions s'affranchissent des résultats des études prévisionnelles.

Hélène Buisson-Fenet helene.buissonfenet@ens-lyon.fr ENS de Lyon, Triangle

Éric Verdier eric.verdier@univ-amu.fr Aix-Marseille université, LEST, CNRS

\section{NOTES}

1 Son développement s'est notamment appuyé sur une régulation de branche conduisant les organisations patronales à conclure avec l'État des conventions pour développer, avec son aide, la formation professionnelle des salariés ainsi que les formations en alternance des jeunes (Méhaut, 2008).

2 Le ministère du Travail inscrit la formation professionnelle, notamment l'apprentissage, dans le giron de la politique de l'emploi tandis que le ministère de l'Éducation nationale fait de la formation des jeunes une composante de la politique éducative. Parfois problématique, cette répartition des compétences porte l'empreinte d'une histoire faite de rivalités inter-administratives (Charlot \& Figeat, 1985).

3 On note par exemple le développement des services d'études et de statistiques des ministères de l'Éducation nationale et du Travail, érigés l'un et l'autre en directions d'administration centrale à la fin des années 1980 et au début des années 1990.

4 Initialement limité à la formation des jeunes (16-25 ans), il est étendu en 2002 à la formation continue des adultes.

5 Document préparatoire au nouveau PRDF, conseil régional PACA, mimeo, 2006.

6 Identifiant 93 familles rassemblées en 22 domaines professionnels, cette nomenclature permet de croiser les informations relatives à la formation, au marché du travail et à l'emploi ; ainsi, pour chaque famille sont recensés les diplômes de l'Education nationale, des ministères du Travail, des Affaires sanitaires et sociales, de la Jeunesse et des Sports (DRDJS), de l'Agriculture etc., pour appliquer cette approche, par exemple, aux statistiques relatives aux entrées en emploi.

7 C'est précisément pour prévenir les défaillances de coordination entre acteurs publics que la déclaration commune de l'Association des Régions de France et de la présidence de la République en date du 10 septembre 2012 prévoit que les Régions piloteront l'évolution de la carte des formations en vue, en particulier, de renforcer la lutte contre le " décrochage " scolaire : la configuration institutionnelle précise n'est pas, à ce jour, arrêtée tant les arbitrages politiques s'avèrent difficiles à rendre.

8 Par exemple : 1. Quel est le panorama actuel des emplois de votre bassin, par secteur d'activité et par domaine de métier ? 2. Quelles sont les activités structurantes ? 3. Quelle est l'évolution des emplois? 4. Quels sont les domaines de métier en difficulté de recrutement ? 5. Les flux de sorties de formations : scolaires, apprentis, formations continues jeunes ou adultes ? 6. Les flux de formations sont-ils adaptés aux besoins d'emplois du bassin ? 7. Quelles opportunités d'emplois possibles pour le projet de formation de la personne? (voir le site du PRAO : www.prao.org).

9 Les syndicats d'enseignants ont fait valoir que le document transmis au Conseil académique de l'Éducation nationale (CAEN) n'était pas conforme aux exigences réglementaires du code de l'éducation, dans la mesure où il n'abordait que les formations professionnelles et technologiques. Ce manquement a justifié qu'on délaisse le schéma prévisionnel. 


\section{BIBLIOGRAPHIE}

AFFICHARD J. (1976). «Bilan de l'emploi par profession ». Économie et Statistique, n॰ 81-82, p. 45-55.

BEL M. \& DUBOUCHET L. (2004). La décentralisation de la formation professionnelle : un processus en voie d'achèvement? La Tour d'Aigues : Éd. de l'Aube.

BEL M., GÉRARD-VARET L.-A. \& VERDIER É. (2000). "L'évolution des modes de construction de l'offre de formation professionnelle ». In Comité de coordination des programmes régionaux d'apprentissage et de formation professionnelle continue, Évaluation des politiques régionales de formation professionnelle 1997-1999. Vol I : Rapports. Paris : La Documentation française, p. 183-215.

BERTHET T., DECHEZELLES S., GAIN R. \& SIMON V. (2010). "La place des dynamiques territoriales dans la régulation de l'orientation scolaire ». Formation emploi, no 109, p. 37-52.

CARO P. \& HILLAU B. (1997). " La logique dominante des publics scolaires. Offre de formations et environnement local ». Formation emploi, no 59, p. 87-103.

CESR RHÔNE-ALPES [Conseil économique, social et environnemental de Rhône-Alpes] (2004). L'espace régional comme espace de dialogue et de négociation sociale. Rapport no 2004-02. En ligne : http://www. cesdefrance.fr/doc.php?id_doc=1192 (consulté le 5 avril 2012).

CHARLOT B. \& FIGEAT M. (1985). Histoire de la formation des ouvriers (1789-1984). Paris : Minerve.

DESROSIÈRES A. (2003). "Historiciser l'action publique : l'État, le marché et les statistiques ". In P. Laborier \& D. Trom (dir.), Historicités de l'action publique. Paris : PUF, p. 207-221.

DESROSIĖRES A. (2008). « Le territoire et la localité. Deux langages statistiques ». In A. Desrosières, Gouverner par les nombres. Paris: Presses de l'École des Mines, p. 84-102.

FOURQUET F. \& MURARD L. (1976). Les équipements $d u$ pouvoir : villes, territoires et équipements collectifs. Paris : Union générale d'éditions.

FOURQUET F. (1983). Les comptes de la puissance. Paris : Recherches.

FREYSSINET J. (1991). Pour une prospective des métiers et des qualifications. Rapport du groupe de travail, Commissariat général du plan. Paris : La Documentation française.
GAUDIN J.-P. (1999). Gouverner par contrat. Paris : Presses de Sciences Po.

JOBERT A. (2002). Les espaces de la négociation collective, branches et territoires. Toulouse : Octarès.

HEALY A. \& VERDIER É. (2010). " Dispositifs de connaissance et action publique en région : les Observatoires régionaux de l'emploi et de la formation. Provence-Alpes-Côte d'Azur versus Rhône-Alpes ». Revue française de socio-économie, n 5, p. 141-164.

INSEE (1976). «Offre et demande d'emploi par profession au cours du 7e Plan ». Économie et Statistique, n॰ 81-82.

MÉHAUT P. (2008). «Formation tout au long de la vie ». In A. van Zanten (dir.), Dictionnaire de l'éducation. Paris : PUF, p. 343-347.

MÉHAUT P. \& VERDIER É. (2009). « Recomposition de l'action publique et émergence de nouvelles scènes de négociation sociale ? L'exemple de la formation professionnelle ». In L. Duclos, G. Groux \& O. Mériaux (dir.), Les nouvelles dimensions du politique : régulations professionnelles et régulations sociales. Paris : LGDJ, p. 201-216.

MISSION D'AUDIT DE MODERNISATION (2006). Rapport sur la carte de l'enseignement professionnel. Paris : Ministère de l'Éducation nationale, Inspection générale des Finances, Inspection générale de l'administration de l'Éducation nationale et de la recherche, Inspection générale de l'Éducation nationale.

PONS X. (2012). « Les statistiques scolaires : vers une science du gouvernement régional ? ". In E. Bornand M. Mespoulet \& É. Verdier, Les politiques de la mesure. L'analyse comparée des indicateurs dans la conduite de l'action régionale. Paris : Karthala ; Aix-en-Provence : Sciences Po Aix, p. 111-127.

SUTEAU M. (1999). Une ville et ses écoles. Nantes, 18301940. Rennes: Presses universitaires de Rennes.

TANGUY L. (2002). « La mise en équivalence de la formation avec l'emploi dans les IVe et Ve Plans (1962-1970) ». Revue française de sociologie, vol. 43, n॰ 4, p. 685-709.

TROGER V. (2008). "Enseignements techniques et professionnels ». In A. van Zanten (dir.), Dictionnaire de l'éducation. Paris : PUF, p. 275-279.

VERDIER É. \& VION A. (2005). " Mix of contracts or contractual mix-up? The coordination of public intervention on access to work in France ». International Journal of Public Policy, no 1-2, p. 161-184. 